\title{
A SHORT COMPUTATION OF THE NORMS OF FREE CONVOLUTION OPERATORS
}

\author{
WOLFGANG WOESS
}

\begin{abstract}
Akemann and Ostrand in 1976 gave a formula for the norms of free convolution operators on the $L^{2}$-space of a discrete group. Using random walk techniques and generating functions, a short and elementary computation of this formula is given.
\end{abstract}

1. Introduction and statement of results. Let $\mathbf{G}$ be a discrete group with unit element $e$ and let $\alpha$ be a complex-valued function on $\mathbf{G}$ supported by a finite set $X$ which has the Leinert property (see [1] for the definition, resp. Lemma 1 below). The main result of the-by now classical-article [1] by Akemann and Ostrand is the following:

THEOREM 1.

$$
\|\alpha\|=\min \left\{2 t+\sum_{x \in X}\left(\sqrt{t^{2}+|\alpha(x)|^{2}}-t\right) \mid t \geqslant 0\right\},
$$

where $\|\alpha\|$ denotes the norm of $\alpha$ as a (left) convolution operator on $L^{2}(\mathbf{G})$ (called "free" operator in [1]).

The aim of this paper is to give a short and elementary proof of this theorem. From the prerequisites of [1] only the following result is used without proof.

LeMma 1. $X \subseteq \mathbf{G}$ has the Leinert property iff $X=y \cdot(Y \cup\{e\})$, where $Y$ is a free set in $\mathbf{G}$ and $y \in X$.

Indeed, $X$ has the Leinert property if and only if there are no nontrivial relations among the elements of $y^{-1} X$ for any fixed $y \in X$ or, equivalently, if and only if $X$ is a left translate of a subset $Y \cup\{e\}$ of $\mathbf{G}$, where $Y$ has no nontrivial relations.

Let $\check{\alpha}(x)=\overline{\alpha\left(x^{-1}\right)}$ for $x \in \mathbf{G}$. $\check{\alpha}$ gives the adjoint convolution operator. We use the formula $\|\alpha\|=\|\mu\|^{1 / 2}$, where $\mu=\check{\alpha} * \alpha$. Let $\mu^{(n)}$ denote the $n$th convolution power of $\mu, \mu^{(0)}=\delta_{e}$.

LEMMA 2. $\|\mu\|=\lim _{n \rightarrow \infty} \mu^{(n)}(e)^{1 / n}$.

Received by the editors August 10, 1984.

1980 Mathematics Subject Classification. Primary 22D25; Secondary 20E05, 43A15, 60J15.

Key words and phrases. Norm of a convolution operator, Leinert property, free group, alternating random walk. 
Thus $\|\alpha\|$ is the inverse of the radius of convergence $r$ of the Taylor series

$$
G(z)=\sum_{n=0}^{\infty} \mu^{(n)}(e) z^{2 n} \quad(z \in \mathbf{C}) .
$$

We shall prove the following result:

THEOREM 2. $G(z)=P(z G(z))$, where

$$
P(t)=1+\frac{1}{2} \sum_{x \in X}\left(\sqrt{1+4|\alpha(x)|^{2} t^{2}}-1\right) \quad(t \in \mathbf{C}) .
$$

As $G(z)$ has positive real coefficients, $r$ is its smallest positive singularity. The same technique as in [3] yields

Corollary 1. $r^{-1}=\inf \{P(t) / t \mid t>0\}$.

If $X$ has more than two elements (the interesting case), then the infimum is a minimum; otherwise, it is attained as $t \rightarrow \infty$. The result of Corollary 1 can be easily transformed into the formula of Theorem 1.

\section{Proofs.}

Proof of Lemma 2. Let $|\alpha|(x)=|\alpha(x)|$ and $|\mu|=|\check{\alpha}| *|\alpha|$. By Lemma $1, \mu^{(n)}(e)$ is the sum over all products

$$
\check{\alpha}\left(x_{i_{1}}^{-1} y^{-1}\right) \alpha\left(y x_{i_{2}}\right) \cdots \check{\alpha}\left(x_{i_{2 n-1}}^{-1} y^{-1}\right) \alpha\left(y x_{i_{2 n}}\right),
$$

where $x_{i_{1}}, \ldots, x_{i_{2 n}} \in Y \cup\{e\}$ and $x_{i_{1}}^{-1} x_{i_{2}} \cdots x_{i_{2 n-1}}^{-1} x_{i_{2 n}}=e$. As $Y$ is a free set, there must be a bijection between the $x_{i j}$ with even index $j$ and those with odd index. Therefore the product in (2) is equal to the corresponding product in the sum that gives $|\mu|^{(n)}(e)$. This yields

$$
\mu^{(n)}(e)=|\mu|^{(n)}(e)
$$

This gives

$$
\begin{aligned}
|\mu|^{(n)}(e)^{1 / n} & =\mu^{(n)}(e)^{1 / n}=\left\langle\mu^{(n)} * \delta_{e}, \delta_{e}\right\rangle^{1 / n} \\
& \leqslant\left\|\mu^{(n)}\right\|^{1 / n}=\|\mu\| \leqslant\|\mu \mid\| .
\end{aligned}
$$

On the other hand, as $X$ is finite, $[2,4]$ yield

$$
\||\mu|\|=\lim _{n \rightarrow \infty}|\mu|^{(n)}(e)^{1 / n}
$$

and, by another application of (3), the lemma is proved.

Thus we have also proved Theorem III G of [1]. If $\beta=c \alpha, c>0$ and $\nu=\not \beta * \beta$ then it is clear that $\nu^{(n)}=c^{2 n} \mu^{(n)}$. This, Lemma 1, (3) and the finiteness of $X$ justify the following assumptions for the proof of Theorem 2 (without loss of generality).

Assumptions. (i) $\mathbf{G}=\mathbf{F}_{s}$ is the free group on $Y=\left\{x_{1}, \ldots, x_{s}\right\}$, and the support of $\alpha$ is $X=\{e\} \cup Y$.

(ii) $\alpha$ is a probability distribution, i.e., $\alpha(e)=\alpha_{0}, \alpha\left(x_{i}\right)=\alpha_{i}, i=1, \ldots, s$, where $\alpha_{i}>0$ (real) and $\sum_{i=0}^{s} \alpha_{i}=1$. 
Now consider a sequence of $\mathbf{G}$-valued random variables $X_{n}, n=0,1,2, \ldots$, constituting a Markov process with transition probabilities

$$
\operatorname{Pr}\left[X_{n+1}=y \mid X_{n}=x\right]= \begin{cases}\check{\alpha}\left(x^{-1} y\right) & \text { if } n \text { is even, } \\ \alpha\left(x^{-1} y\right) & \text { if } n \text { is odd. }\end{cases}
$$

This gives an "alternating random walk" on the homogeneous tree of degree $2 s$ that represents $\mathbf{F}_{s}$. We have $\mu^{(n)}(e)=\operatorname{Pr}\left[X_{2 n}=e \mid X_{0}=e\right]$. Let $p^{(2 n-1)}=\operatorname{Pr}\left[X_{2 n}=e \mid X_{1}\right.$ $=e]$ and $H(z)=\sum_{n=1}^{\infty} p^{(2 n-1)} z^{2 n-1}$. Besides $\mu^{(n)}(e)$ and $p^{(2 n-1)}$ we need the following "taboo probabilities" and their generating functions for $i=1, \ldots, s$ :

$$
\begin{aligned}
& f_{i}^{(n)}=\operatorname{Pr}\left[X_{n}=e ; X_{m} \neq e \text { for } m=1, \ldots, n-1 ; X_{1}=x_{i}^{-1} \mid X_{0}=e\right], \quad f_{i}^{(0)}=0, \\
& a_{i}^{(2 n)}=\operatorname{Pr}\left[X_{2 n+1}=e ; X_{m} \neq x_{i} \text { for } m=2, \ldots, 2 n \mid X_{1}=e\right], \quad a_{i}^{(0)}=1, \\
& b_{i}^{(2 n-1)}=\operatorname{Pr}\left[X_{2 n-1}=e ; X_{m} \neq x_{i} \text { for } m=1, \ldots, 2 n-2 \mid X_{0}=e\right], \\
& F_{i}(z)=\sum_{n=0}^{\infty} f_{i}^{(2 n)} z^{2 n}, \quad A_{i}(z)=\sum_{n=0}^{\infty} a_{i}^{(2 n)} z^{2 n}, \quad B_{i}(z)=\sum_{n=1}^{\infty} b_{i}^{(2 n-1)} z^{2 n-1} .
\end{aligned}
$$

Furthermore, let $f^{(n)}=\sum_{i=1}^{s} f_{i}^{(n)}$ and $F(z)=\sum_{i=1}^{s} F_{i}(z)$. Note that $f_{i}^{(2 n-1)}=0$ for all $n \geqslant 1$, as the $(2 n-1)$ st step cannot lead from $x_{i}^{-1}$ to $e$ with positive probability.

LEMMA 3. (a) $G(z)=1+F(z) G(z)+\alpha_{0} z H(z)$,

(b) $H(z)=F(z) H(z)+\alpha_{0} z G(z)$,

(c) $A_{i}(z)=1+\left(F(z)-F_{i}(z)\right) A_{i}(z)+\alpha_{0} z B_{i}(z)$,

(d) $B_{i}(z)=F(z) B_{i}(z)+\alpha_{0} z A_{i}(z)$

(e) $F_{i}(z)=\alpha_{i}^{2} z^{2} A_{i}(z), i=1, \ldots, s$.

Proof. This is obvious from the following relations

$$
\mu^{(n)}(e)=\sum_{k=0}^{n} f^{(2 k)} \mu^{(n-k)}(e)+\alpha_{0} p^{(2 n-1)} \quad \text { for } n \geqslant 1, \quad \mu^{(0)}(e)=1 .
$$

Here and in (6), (7) and (8) the summation on the right is taken over all possible instants of first return to $e$, which are $2 k(k=0, \ldots, n$, resp. $n-1)$ and 1 . In (6) and (7) we use the fact that the symmetry between $\alpha$ and $\check{\alpha}$ gives us

$$
\begin{aligned}
f_{i}^{(2 k)} & =\operatorname{Pr}\left[X_{2 k+1}=e ; X_{m} \neq e \text { for } m=2, \ldots, 2 k ; X_{2}=x_{i} \mid X_{1}=e\right] . \\
p^{(2 n-1)} & =\sum_{k=0}^{n-1} f^{(2 k)} p^{(2 n-2 k-1)}+\alpha_{0} \mu^{(n-1)}(e) \\
a_{i}^{(2 n)} & =\sum_{k=0}^{n}\left(f^{(2 k)}-f_{i}^{(2 k)}\right) a_{i}^{(2 n-2 k)}+\alpha_{0} b_{i}^{(2 n-1)} \quad \text { for } n \geqslant 1, a_{i}^{(0)}=1 . \\
b_{i}^{(2 n-1)} & =\sum_{k=0}^{n-1} f^{(2 k)} b_{i}^{(2 n-2 k-1)}+\alpha_{0} a_{i}^{(2 n-2)} \quad \text { for } n \geqslant 1 . \\
f_{i}^{(2 n)} & =\alpha_{i}^{2} a_{i}^{(2 n-2)} \quad \text { for } n \geqslant 1, f_{i}^{(0)}=0 .
\end{aligned}
$$

In (9) we have used the fact that the transitions from $x$ to $y$ and from $e$ to $x^{-1} y$ have the same probability.

Proof of Theorem 2. Let $F_{0}(z)=\alpha_{0}^{2} z^{2} /(1-F(z))$. From (a) and (b) we obtain

$$
G(z)=1 /\left(1-F(z)-F_{0}(z)\right) \text {. }
$$


Replacing $1-F(z)$ by $\alpha_{0}^{2} z^{2} / F_{0}(z),(10)$ gives a quadratic equation for $F_{0}(z)$ which has the solution

$$
F_{0}(z)=\left(\sqrt{1+4 \alpha_{0}^{2} z^{2} G(z)^{2}}-1\right) / 2 G(z) .
$$

Among the two solutions this is the proper one because $G(0)=1$ and $F_{0}(0)=0$. (c) and (d) yield $A_{i}(z)=1 /\left(F_{i}(z)+1 / G(z)\right)$, and by (e),

$$
F_{i}(z)=\alpha_{i}^{2} z^{2} /\left(F_{i}(z)+1 / G(z)\right)
$$

which gives, like (11),

$$
F_{i}(z)=\left(\sqrt{1+4 \alpha_{i}^{2} z^{2} G(z)^{2}}-1\right) / 2 G(z) \text { for } i=1, \ldots, s .
$$

If we write (10) in the form $G(z)=1 /\left(1-\sum_{i=0}^{s} F_{i}(z)\right)$ and use (11) and (13), we then obtain the proposed equation for $G(z)$.

Proof of Corollary 1. This is proved exactly like Proposition 3 in [3]. For the sake of completeness, the lines of the proof are indicated.

By Theorem 2,w $=G(z)$ solves $\mathscr{F}(z, w)=0$, where

$$
\mathscr{F}(z, w)=P(z w)-w,
$$

and this function is analytic for positive $z, w$. The radius of convergence $r$ is the smallest positive singularity of $G(z)$.

For positive real $t$, the convex curve $y=P(t)$ approaches the asymptote $y=$ $\left(\sum_{x \in X}|\alpha(x)|\right) t-(s-1) / 2$. For $0<z<r, G(z)$ is positive real and can be illustrated as the ordinate of the point of intersection of the line $y=(1 / z) t$ with $y=P(t)$ in the real $(t, y)$-plane. If there are two points of intersection, by continuity, the proper one is the one closer to the origin.

If $s=0$ or $s=1$, then for each positive $z<\left(\sum_{x \in X}|\alpha(x)|\right)^{-1}$ we find exactly one solution, and the angle of intersection is nonzero. By the implicit function theorem, applied to (14), the solution $G(z)$ is analytic at $z$. For larger positive real $z$, there is no real solution at all, hence

$$
r^{-1}=\sum_{x \in X}|\alpha(x)|=\lim _{t \rightarrow \infty}(P(t) / t)=\inf \{P(t) / t \mid t>0\}, \quad s=0,1 .
$$

If $s \geqslant 2$, then the asymptote passes below the origin, and there is a unique positive solution $\theta$ of the equation $t P^{\prime}(t)=P(t)$. As above, for positive $z<\theta / P(\theta)$ we can find an analytic solution $G(z)$ which is positive real, whereas for larger $z$ there is no such solution. Thus

$$
r^{-1}=P(\theta) / \theta=\min \{P(t) / t \mid t>0\}, \quad s \geqslant 2 .
$$

\section{REFERENCES}

1. Ch. A. Akemann and Ph. A. Ostrand, Computing norms in group $C^{*}$-algebras, Amer. J. Math. 98 (1976), 1015-1047.

2. Ch. Berg and J. P. R. Christensen, Sur la norme des opérateurs de convolution, Invent. Math. 23 (1974), 173-178.

3. P. Gerl and W. Woess, Local limits and harmonic functions for nonisotropic random walks on free groups, Z. Wahrsch. Verw. Gebiete (to appear).

4. H. Kesten, Symmetric random walks on groups, Trans. Amer. Math. Soc. 92 (1959), 336-354.

InSTitut FỨ Mathematik und ANGewandte Geometrie, Montanuniversität, A-8700 LeOBEn, AUSTRIA 